http://jmscr.igmpublication.org/home/ ISSN (e)-2347-176x ISSN (p) 2455-0450 crossref DOI: https://dx.doi.org/10.18535/jmscr/v8i3.38

\title{
A Giant Chondrosarcoma Born on Primary Osteochondroma - A Rare Case Report
}

\author{
Authors \\ Dr Vinay Kumar.J.Rajendra ${ }^{1 *}$, Dr Sruthy Harshan ${ }^{2}$ \\ ${ }^{1}$ Associate Professor and Consultant Surgical Oncologist, Department of Surgical Oncology, K.S. Hegde \\ Medical Academy, Deralakatte, Mangalore \\ ${ }^{2}$ Junior Resident, Department of General Surgery, K.S.Hegde Medical Academy, Deralakatte, Mangalore \\ *Corresponding Author \\ Dr Vinay Kumar J Rajendra
}

\begin{abstract}
Background: Chondrosarcoma is a rare malignant tumor of the bone. Secondary chondrosarcoma originating from a primary osteochondroma is extremely rare and the data show that the reported incidence of shoulder osteochondroma is very low. We report the case of a 28 yr old male patient with Chondrosarcoma secondary to malignant transformation of an Osteochondroma of left shoulder. The patient was diagnosed with left shoulder Osteochondroma 7 years back for which tumour excision was done. He currently presented with a $20 \times 20 \times 15 \mathrm{~cm}$ swelling over the left scapular region since two years. MRI of Shoulder revealed a large expansile altered signal intensity lesion arising from the left scapula. Biopsy showed roughly cartilaginous appearance and was diagnosed to be well differentiated Chondrosarcoma. Patient was therefore a candidate for a large resection. HRCT thorax was done which showed no parenchymal lung lesion. Intra-operatively, Glenoid capsule, superior and lateral cord of Brachial plexus was involved. Histopathology showed secondary low grade Chondrosarcoma of scapula with all resected margins free of tumour. He is now at 1 year of surgical resection, and is doing well with restriction in movements at shoulder joint.
\end{abstract}

Keywords: Chondrosarcoma, osteochondroma, scapula.

\section{Introduction}

Primary chondrosarcoma is the third most common primary tumour of the bone; however secondary chondrosarcoma arising from a primary osteochondroma is an extremely rare entity. ${ }^{1}$

Prior data reveals that reported incidence of osteochondromas arising from shoulder is extremely rare. Most of the chondrosarcoma grow slowly and rarely metastasise, and they have an excellent ${ }^{2}$ prognosis after adequate surgery. However most of them are chemo or radio-resistant. ${ }^{3}$ Slow growth and late recurrence require long-term follow-up to understand the clinical course.

\section{Case Report}

We report the case of a $28 \mathrm{yr}$. old male patient, with chondrosarcoma secondary to malignant transformation of an osteochondroma of the left shoulder.

The patient was diagnosed with left shoulder osteochondroma 7 years back, owing to which tumor excision was done. He currently presented 
touswith a $20 \times 20 \times 15 \mathrm{~cm}$ swelling over the left scapular region since 2 years.

On examination, there was a hard immobile swelling, with irregular surface and well defined borders, fixed to the underlying bone. Distal pulses were felt ; with no sensory motor deficits. The range of abduction (0-40 degree) and internal rotation (030 degrees) was grossly affected.

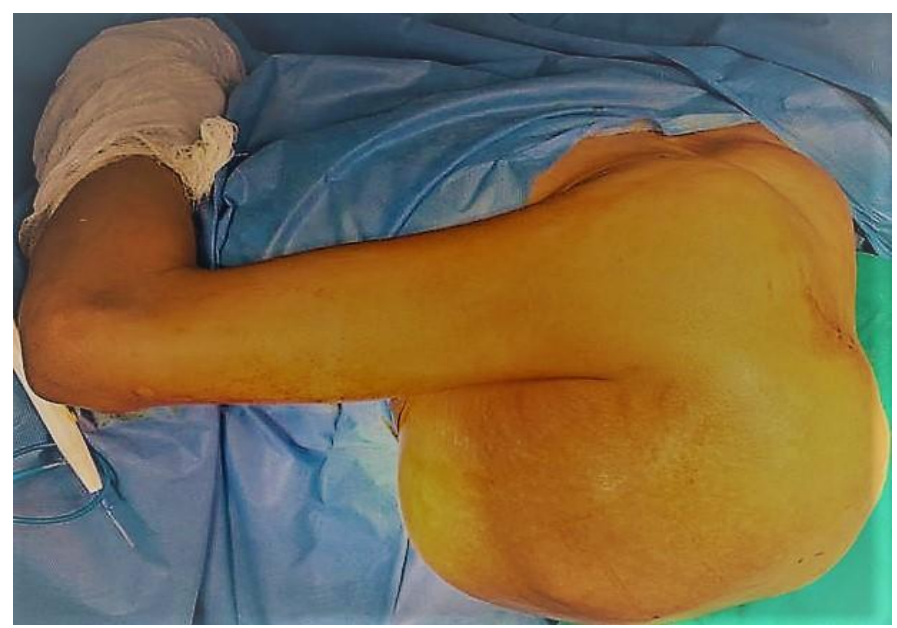

Fig :1 Hard immobile swelling left shoulder .

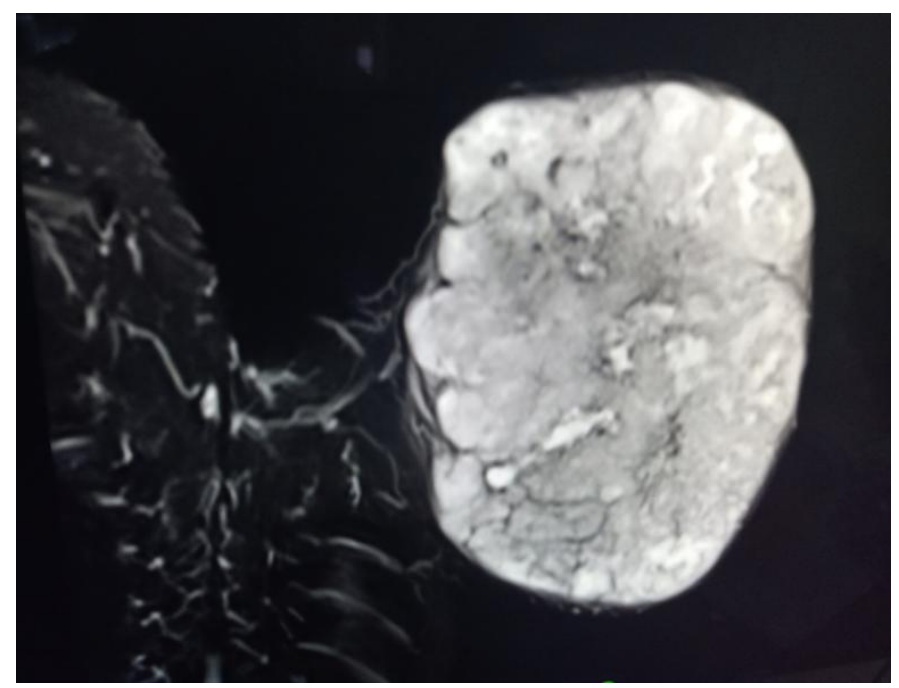

Fig: 2 MRI showing irregular calcification

MRI of shoulder revealed a large expansile altered signal intensity lesion arising from the left scapula with sparing of the glenoid, irregular calcifications were noted. A core needle biopsy was performed, which showed that the tissue had a roughly cartilaginous appearance.

The diagnosis of well differentiated chondrosarcoma of left scapula was made. Routine tests were done to assess the pulmonary function, HRCT thorax showed no parenchymal lung lesions.

The patient was therefore a candidate for large resection. On the basis of this diagnosis, no adjuvant treatment was administered. $\mathrm{He}$ was advised regarding the surgical options of left hindquarter amputation or total excision of scapula and head of humerus with reconstruction. However patient was not willing for the same.

He underwent left total scapulectomy. Intra operatively glenoid capsule, superior and lateral cord of brachial plexus were involved.

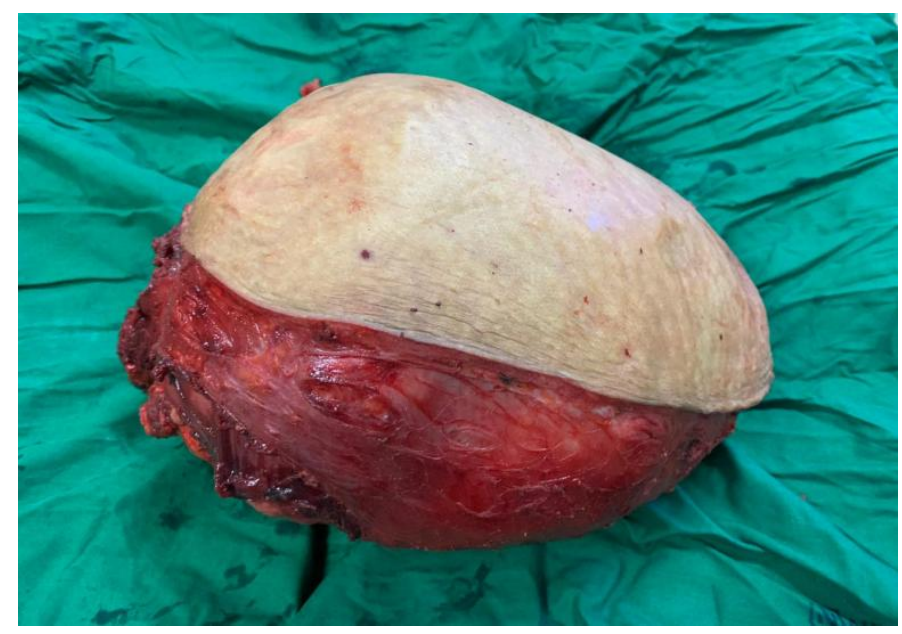

Fig:3 Gross appearance of the tumor.

Histopathology revealed low grade chondrosarcoma of scapula, however resected margins were not free of tumor. He is now at 1 year of surgical resection, with movements at shoulder joint not preserved.

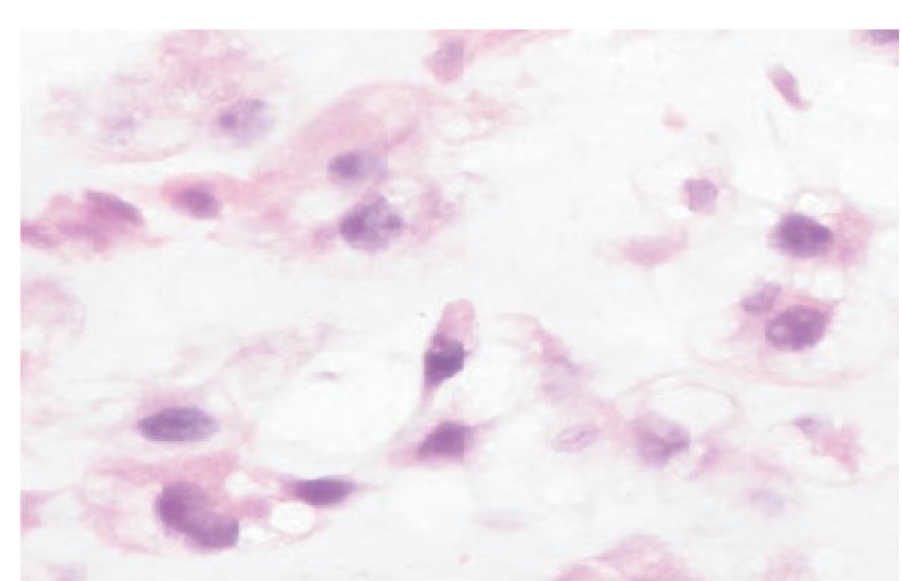

Fig:4 Low grade Chondrosarcoma

\section{Discussion}

Although, osteochondroma is not a true neoplasm, it is the most frequent tumor of bone. Similarly, 
multiple exostoses are "the most common of the systematized anomalies of skeletal development encountered clinically." Chondrosarcoma is considered as a rare complication of osteochondroma. ${ }^{3}$

Chondrosarcoma arising in osteochondroma is usually noticedin younger patients. The most frequent signis usually a mass, of long duration. In thecase of an adult patient with osteochondroma, recent mass progressively increasing in size is a worrisome feature ${ }^{3}$

Prior studies have shown that, there is always apredilection for tumor occurrence in flat bones. Large size and typical pattern of cartilage calcification were useful diagnostic clues. ${ }^{3}$

The criteria used for the diagnosis of secondary chondrosarcoma are increased cellularity, binucleate cells and nuclear atypia. The diagnosis of welldifferen- tiated (Grade 1) chondrosarcomas is extremely difficult.

Inadequate surgical removal almost always will result in recurrence. Primary resection or amputation appears to be the treatment of choice. ${ }^{4}$

\section{Conclusions}

It is generally believed that chondrosarcoma is relatively chemo- and radiotherapy resistant due to the extracellular matrix invasion, poor vascularity and low percentage of dividing cells.

Preservation of the functional capacities of the involved limb along with a complete removal of the tumour is considered as the most important criterion in the surgical management of the tumor. ${ }^{4}$

\section{References}

1. Alessandro Franchi. Epidemiology and classification of bone tumors. Clinical Cases in Mineral and Bone Metabolism 2012; 9(2): 92-5.

2. Tsutomu Akahane. Differentiated chondrosarcoma arising in a solitary osteochondroma with leiomyosarcomatous component: a case report, Arch Orthop Trauma Surg (2008) 128:951-953.
3. Hauben EI, Arends J, Vandenbroucke JP, et al. Multiple primary malignancies in osteosarcoma patients. Incidence and predictive value of osteosarcoma subtype for cancer syndromes related with osteosarcoma.

4. Hameetman L, Bovée JV, Taminiau AH, et al. Multiple osteochondromas: clinicopathological and genetic spectrum and suggestions for clinical management. Hered Cancer Clin Pract 2004;2:161-73. 